\title{
Technical Change and Applications of Dynamic Duality to Agriculture: Reply
}

\author{
Wayne H. Howard and C. Richard Shumway
}

Larson has made three basic points in his comment on the incorporation of technical change in dynamic duality models: $(a)$ appending a time trend onto the empirical model to account for technical change is inconsistent with an autonomous theoretical model, (b) a current value function $V$ discounted to time $t_{o}$, $0<t_{0}<T$, should not be confused with a value function $J$ discounted to time $t=0$, and $(c)$ the firm's expectations of embodied technical change should be explicitly formulated in the theoretical model. The first issue focused on the Vasavada and Chambers paper, and the last two issues were addressed to the Howard and Shumway paper. We will respond to each of these points in order.

First, Larson is correct that Vasavada and Chambers (and several other dynamic duality studies) have maintained an autonomous theoretical model but estimated a nonautonomous empirical model. The bridge between the two models is the assumption of static ex ante technology expectations on the part of producers with ex post awareness of technical change on the part of the analyst. While this assumption has seldom been made explicit in research reports and may appear inconsistent, its practical importance is often minor. For example, although we reported results in our $A J A E$ article only for the nonautonomous theoretical and empirical model, we also estimated the parameters for the autonomous theoretical-nonautonomous empirical model. There were no differences between the two models in any of the test conclusions and only minor differences in the estimated rateof-adjustment parameters.

Second, Larson's equation (11) is essentially the same as our equation ( $\left.3^{\prime}\right)$ except that he defines a current value function $V$, discounted to time $t_{o}, 0<$ $t_{o}<\infty$, while we define an initial value function $J$, discounted to time $t=0$. Larson alleges that the discounting period alters the interpretation of the

Wayne H. Howard and C. Richard Shumway are, respectively, an assistant professor, Department of Agricultural Economics and Business, University of Guelph, and a professor, Department of Agricultural Economics, Texas A\&M University. parameter estimates. However, the output supply, variable input demand, and investment equations are all unaffected by the discounting period. Thus, the interpretation of the estimated output supply and variable input demand equations, the rate-ofadjustment parameters and associated marginal values of investment in quasi-fixed inputs are the same whether one assumes the decision maker acts at time $t=0$ or $t=t_{o}, 0<t_{o}<\infty$.

Finally, Larson correctly notes that we did not explicitly discuss how embodied technical change was included in our theoretical model. The implicitly maintained assumption was Larson's third alternative, i.e., that firms have static embodied technology expectations. Although this assumption may seem counterintuitive given our maintained hypothesis of nonstatic disembodied technology expectations, Larson's other alternatives also carry their own dilemmas. His first alternative was that firms could forecast future-embodied technical change with perfect accuracy (an impossibility), and his second was that firms expect disembodied technical change but actually experience embodied technical change. Each alternative maintains some conceptual baggage, the implications and empirical seriousness of which have not been fully explored.

We are appreciative of Larson's identification of these issues and for the opportunity to enter into a dialogue on some of their theoretical and practical aspects. We would also note a printing error of some consequence near the bottom of the second column of p. 838 of our paper. It should read $F_{\dot{z}}<0$.

\section{References}

Howard, W. H., and C. R. Shumway. "Dynamic Adjustment in the U.S. Dairy Industry." Amer. J. Agr. Econ. 70(1988):837-47.

Vasavada, U., and R. G. Chambers. "Investment in U.S. Agriculture." Amer. J. Agr. Econ. 68(1986):950-60. 